ORI GI NAL

\title{
EVALUACIÓN DE TRES METODOLOGÍAS DE RECUPERACIÓN DE EMBRIONES EN CANINOS UTILIZANDO UN MODELO POSTMORTEM
}

\section{EVALUATI ON OF THREE TECHNI QUES TO RECOVER CANI NE EMBRYOS USING A POST-MORTEM MODEL}

\author{
*Claudia Jiménez E, DVSC, Omar Bustos B, M.Sc. \\ Universidad Nacional de Colombia, Facultad de Medicina Veterinaria y de Zootecnia. Bogotá, \\ Colombia. Correspondencia: cjimeneze@unal.edu.co
}

Recibido: Mayo 12 de 2008; Aceptado: Diciembre 10 de 2008

\section{RESUMEN}

Objetivo. Evaluar tres técnicas de recuperación de embriones, utilizando como modelos de simulación oocitos desnudos introducidos en úteros recuperados post-mortem. Materiales y métodos. Se introdujeron 10 oocitos en el extremo ovárico de cada cuerno. El tratamiento 1 (T1) consistió en realizar una punción a nivel del extremo ovárico de cada cuerno para introducir el medio de lavado. Se incidió la base de cada cuerno para ubicar un catéter TOMCAT 8-Fr, recuperando el medio en una caja de petri. En el tratamiento 2 (T2) se realizó una punción en el extremo ovárico de cada cuerno pero la recuperación de los oocitos se realizó con un catéter de $56 \mathrm{~cm}$ de longitud que se pasó transcervicalmente hasta la base de cada cuerno. En el tratamiento 3 (T3) se utilizó el mismo tipo de catéter por vía transcervical y el lavado y la recuperación se realizó a través del mismo catéter. Resultados. El número de estructuras recuperadas fue de $7.3 \pm 2.4$ (73\%) para el T1; $8.5 \pm 1.9$ (85\%) para el T2 y de $5.8 \pm 2.2(58 \%)$ para el T3. La tasa de recuperación fue similar para T1 y T2, pero significativamente mayores que para el T3 $(p>0.01)$. Conclusiones. Las técnicas que se proponen son menos invasivas y tienen resultados comparables a otros estudios donde realizan histerectomía y laparotomía. Con este tipo de técnicas, se disminuye el trauma generado por incisiones quirúrgicas y la posible generación de adherencias. Adicionalmente permitirían realizar recuperaciones de embriones no quirúrgicas en caninos.

Palabras clave: Embriones, oocitos, recuperación, transferencia, caninos.

\section{ABSTRACT}

Objective. To compare three embryo recovery techniques, using simulation models in which denuded oocytes were introduced in recently recovered uteri. Materials and methods. Ten denuded oocytes were deposited in the ovarian tip of each uterine horn 
using a Tomcat catheter. Technique one (T1) consisted of a puncture made close to the ovarian tip of the uterine horn with a $20 \mathrm{G}$ needle connected to a $20 \mathrm{ml}$ syringe loaded with $20 \mathrm{ml}$ of PBS (flush medium). In the base of each horn, a $1 \mathrm{~cm}$ incision was made to place the Tomcat catheter from which the fluid was recovered into a Petri dish. For technique two (T2) a puncture in the ovarian tip was made as in T1 but the oocytes were recovered with a catheter that was passed through the cervix and the fluid recovered in a Petri dish. In technique three (T3) a catheter was inserted through the cervix and was used for pumping the collection media in and out. Results. The number of structures recovered per horn was $7.3 \pm 2.4(73 \%)$ for $\mathrm{T} 1 ; 8.5 \pm 1.9(85 \%)$ for $\mathrm{T} 2$ and $5.8 \pm 2.2(58 \%)$ for T3. The first two techniques were not significantly different $(p>0.01)$ and showed better recovery rates than the technique used in T3. Conclusions. Canine embryos could be retrieved using a less invasive technique by passing an 8-french catheter through the cervix thereby reducing the damage to the uterus and the possible generation of adhesions.

Key words: Embryos, oocytes, recovery, canine, embryo transfer

\section{NTRODUCCIÓN}

La transferencia de embriones (ET) es una de las técnicas de reproducción asistida más utilizada en el mundo en especies productivas como los bovinos, caprinos y equinos. En caninos esta tecnología aún no está masificada pero podría ser una herramienta útil en la recuperación de especies en vía de extinción como los zorros y a nivel comercial esta técnica ayudaría a mejorar la eficiencia en los criaderos.

En otros mamíferos se han establecido diferentes métodos de recuperación de embriones que van desde la utilización de sondas intrauterinas hasta la práctica de cirugía. En caninos, las investigaciones realizadas hasta el momento, han utilizado dos métodos de recuperación: el lavado del útero realizado por laparotomía o por histerectomía $(1,3)$.

En un trabajo realizado por Tsutsui (3), se evaluó la recuperación de embriones en perras de la raza Beagle por los métodos anteriores. La tasa de recuperación de embriones (TRE) obtenidos 8 a 11 días después de la ovulación, en 16 perras, fue de $97.1 \%$ por el método de extracción, mientras que por laparotomía fue de $42.5 \%$ en 3 perras.

En otro trabajo realizado en zorros silvestres, se utilizaron 8 animales que fueron sacrificados para extraer el útero y luego recuperar los embriones por lavado (1). La tasa de recuperación de embriones de los animales sacrificados 8 a 11 días después del pico de resistencia eléctrica de la vagina, de ocho donadoras en diferentes estados de desarrollo, fue de $66 \%$ basada en un promedio de cuerpos lúteos de 5.5.

Son pocas las investigaciones realizadas en este tema y es por eso importante desarrollar trabajos y estandarizar técnicas que permitan la recuperación de embriones para implementar programas de transferencia de embriones en caninos. Este es el primer paso para establecer biotecnologías que pueden ser útiles en programas de recuperación de fauna silvestre, en el aprovechamiento reproductivo de animales de importancia genética que por alguna dificultad física estaban impedidos para reproducción, en el mejoramiento de la productividad en criaderos, en el intercambio genético entre países y para promover las investigaciones en estas áreas del conocimiento.

En este contexto y con el propósito de brindar un aporte al conocimiento de biotecnologías en reproducción asistida para caninos, se desarrolló el presente trabajo con el objetivo de evaluar y estandarizar metodologías de recuperación de embriones importantes para el desarrollo de protocolos de transferencia de embriones en caninos. 


\section{MATERI ALES Y MÉTODOS}

El trabajo se realizó en el laboratorio de embriones la Facultad de Medicina Veterinaria y Zootecnia de la Universidad Nacional, sede Bogotá.

Material biológico. Para este experimento se emplearon 60 perras criollas adultas, en un rango de peso entre los 18 y $25 \mathrm{~kg}$. Los animales fueron suministrados por Zoonosis (Dirección General de Salud Publica del Ministerio de Protección social de Colombia) después de ser sacrificados dentro de un plan de control poblacional. Para cada tratamiento se emplearon 20 úteros de los animales sacrificados.

Obtención de oocitos. Para evaluar la recuperación de embriones se utilizaron oocitos extraídos de ovarios de perras sacrificadas (Figura 1). Los ovarios se preseleccionaron tratando de evitar ovarios con cuerpos luteos (Figura 1a). A los ovarios se les realizó diferentes incisiones con una cuchilla (Figura 1b), para después ser lavados con solución PBS dentro de una caja de Petri. Posteriormente, se seleccionaron los oocitos bajo un estereoscopio a $100 \mathrm{X}$ y para ser desnudados de manera mecánica con la ayuda de un Vortex ${ }^{\circledR}$ (Figura 1c).

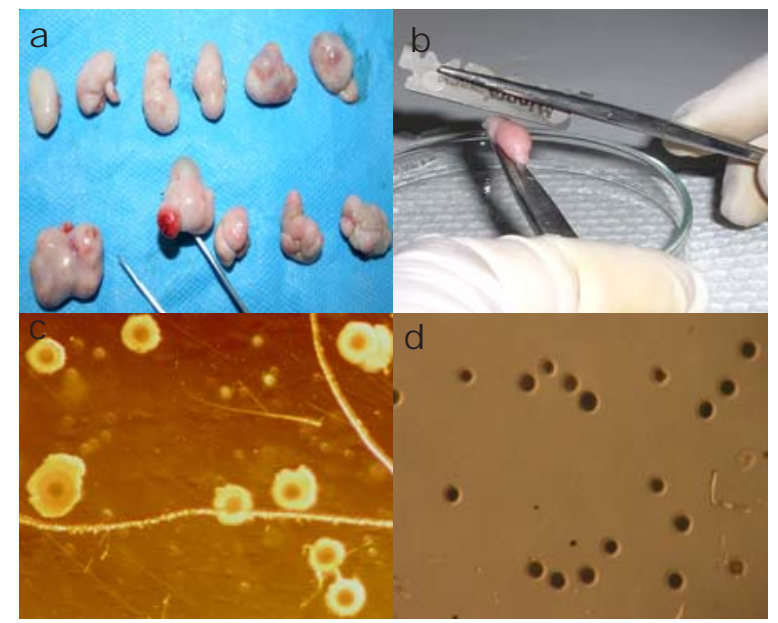

Figura 1. Procedimiento utilizado para la obtención de oocitos de ovarios extraídos de perras sacrificadas en el Instituto de Zoonosis, a. Ovarios seleccionados, b. Sección y lavado de los ovarios para extracción de los oocitios. c. Oocitos seleccionados para ser desnudados por acción mecánica, d. oocitos desnudos para el experimento.
I ntroducción y recuperación de oocitos. Se introdujeron 10 oocitos desnudos (sin capas de cumulus) en cada cuerno de los úteros extraídos de hembras caninas post mortem. La introducción se realizó en el extremo ovárico de cada cuerno del útero. Con una aguja de calibre 20 se perforó el útero para permitir el paso de un catéter TOMCAT 8Fr, el cual contenía los oocitos. Después de introducir el catéter, se vació su contenido en el lumen del cuerno (Figura 2).

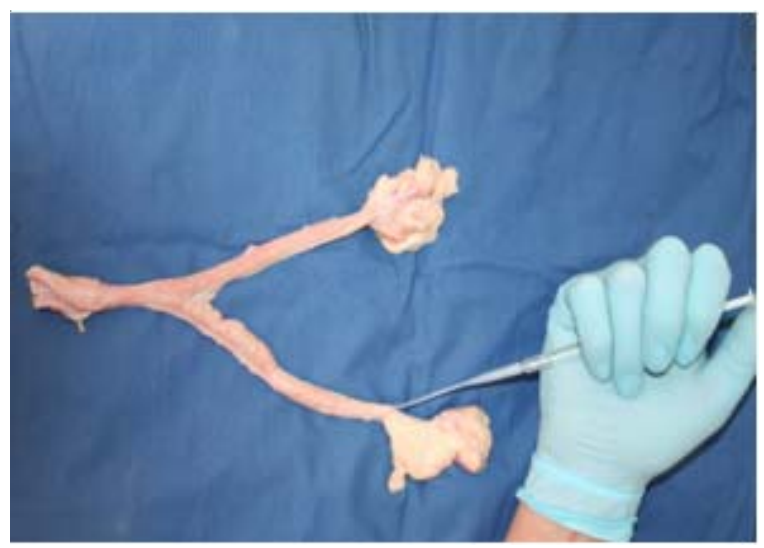

Figura 2. Colocación de 10 oocitos desnudos en el extremo ovárico del cuerno uterino.

Tratamientos. Tratamiento 1 (T1). Se realizó una punción en la parte craneal del cuerno a través de la cual se insertó una aguja calibre 20 ( $\sin$ bisel) y a través de la cual se infundieron $20 \mathrm{ml}$ de lactato de Ringer con $20 \%$ de suero canino. La recuperación de los oocitos se realizó a través de un catéter TOMCAT 8-Fr ubicado en la base del cuerno, para lo cual se debió realizar una incisión de aproximadamente un centímetro con el fin de acomodar la parte ancha del catéter y recuperar los oocitos (Figura 3).

Tratamiento 2 (T2). Se realizó una punción en la parte craneal del cuerno a través de la cual se insertó una aguja calibre 20 ( $\sin$ bisel) y por la cual se infundieron $20 \mathrm{ml}$ lactato de Ringer con $20 \%$ de suero canino. La recuperación de los oocitos se realizó a través de un catéter TOMCAT 8Fr rígido, de $56 \mathrm{~cm}$ de longitud pasado transcervicalmente y ubicado en la base de cada cuerno (Figura 4). 


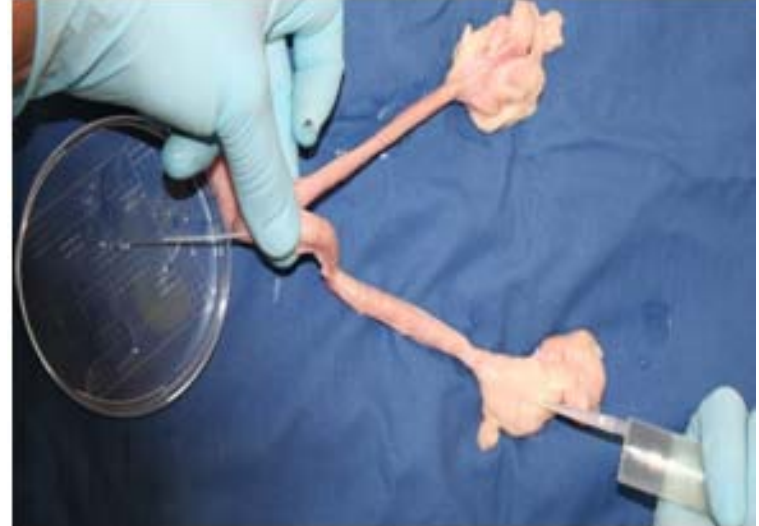

Figura 3. Tratamiento 1 . Se realizaron dos incisiones para recuperar los oocitos. La primera se realizó en el extremo ovárico del cuerno para introducir el medio de lavado. La segunda se realizó en la base de cada cuerno para recuperar los oocitos a través de un catéter Tomcat 8Fr.

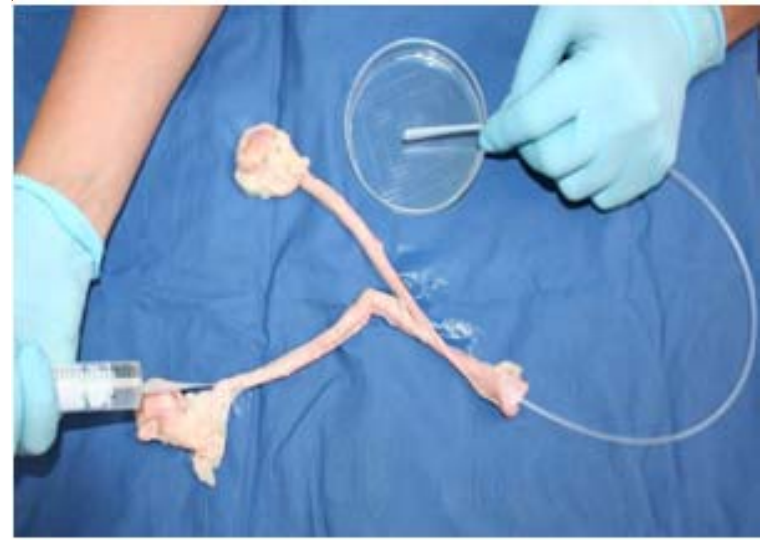

Figura 4. Tratamiento 2 . Se realizó una incisión para recuperar los oocitos. La perforación se realizó en el extremo ovárico del cuerno para introducir el medio de lavado. La recuperación se realizó a través del cervix con un catéter Tomcat 8Fr. Largo

Tratamiento 3 (T3). Por vía vaginal se pasó transcervicalmente un catéter TOMCAT 8Fr, de $56 \mathrm{~cm}$ de longitud, el cual se ubicó en la base de cada cuerno. El medio de lavado se introdujo por el catéter hasta distender los cuernos. Las estructuras se recuperaron a través del catéter, generando un flujo y succionando con la jeringa para permitir la recuperación del líquido depositado en cada cuerno (Figura 5).

Los oocitos recuperados de cada perra, se colocaron en una caja de Petri para ser

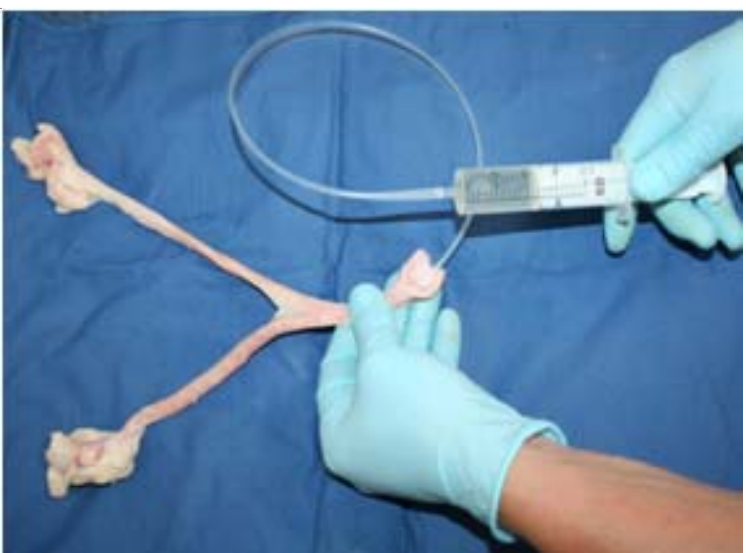

Figura 5. Tratamiento 3. No se realizó ninguna incisión del útero. El medio de lavado se introdujo y los oocitos se recuperaron a través de un catéter Tomcat $8 \mathrm{Fr}$ de $56 \mathrm{~cm}$ de longitud introducido al útero transcervicalmente.

contados y observados en un estereoscopio a 100X.

Parámetros medidos. Tasa de recuperación (TRE): se evaluó el número de oocitos recuperados respecto al número de oocitos transferidos a cada animal.

$$
\text { TRE }=\frac{\text { No oocitos recuperados }}{\text { No de oocitos transferidos }}
$$

Porcentaje de recuperación (\% RE) : se evaluó el porcentaje de oocitos recuperados respecto a los oocitos transferidos.

$\%$ RE $=$ TRE $/ 100$

Evaluación del método. Se evaluó el grado de dificultad en la manipulación de los animales en cada método en una escala de 1 a 5, donde 1 fue el grado de dificultad menor y 5 el grado de dificultad mayor. La misma categorización se hizo para determinar el grado de invasividad.

Análisis estadístico. Los datos obtenidos fueron analizados mediante un análisis de varianza ANOVA, en el programa Statistical Analysis System (SAS) en un modelo completamente al azar.

$Y_{i}=\mu+T_{i}+e_{i}$ 
Donde $\mathrm{i}=1,2,3$, correspondieron a los métodos de lavado.

\section{RESULTADOS}

El tratamiento 2 donde se realizó una punción para lavar el útero y recuperar los oocitos a través de un catéter pasado transcervicalmente tuvo una mejor tasa de recuperación (TR) 0,845, que la de los tratamientos $1($ TR 0,73$)$ y $3($ TR 0,583$)$. La diferencia mínima significativa (dms) $(p<0.05)$ (Tabla 1$)$, solo fue con el tratamiento 3, el cual consistió en el lavado y recuperación de oocitos a través de un catéter ubicado transcervicalmente.

EI T1 donde se realizaba una punción para lavar y se recuperaba por un catéter Tomcat largo fue significativamente mejor $(p<0.05)$ que el T3 (Tabla 1).

Tabla 1. Promedio, Tasa de recuperación (TR) y $\%(\mathrm{R})$ de recuperación de embriones.

\begin{tabular}{lccc}
\hline & T1 & T2 & T3 \\
\hline PROMEDIO & $7.30 \pm 2.4^{\mathrm{s}}$ & $8.45 \pm 1.9^{\mathrm{a}}$ & $5.83 \pm 2.2^{\circ}$ \\
TR & 0.73 & 0.845 & 0.583 \\
$\%$ R & 73 & 84.5 & 58.3 \\
\hline
\end{tabular}

El grado de dificultad fue mayor para el tratamiento T3, donde se introdujo transcervicalmente un catéter pasado desde la vagina y no existio posibilidad de manipular el cérvix directamente; el T2 hubo mayor facilidad ya que la introducción del catéter se realizo manipulando el cervix de manera quirúrgica. El T1, donde se realizo una punción en la punta del cuerno y una incisión en la base del cuerno es netamente quirúrgico; sin embargo, el procedimiento de lavado per se, es relativamente más fácil que los otros dos (Tabla 2).

Tabla 2. Grado de dificultad y de invasión del procedimiento.

\begin{tabular}{lccc}
\hline & T1 & T2 & T3 \\
\hline Grado de dificultad & 2 & 3 & 5 \\
Grado invasión & 5 & 2 & 0 \\
\hline
\end{tabular}

EI T1, donde se realizó cirugía y una incisión en la base de cada cuerno, tiene un grado de invasión mayor que la del tratamiento 2 , donde la laparotoromía si se haría pero no se realizaría la incisión en la base de cada cuerno. Finalmente el T3 tiene el menor grado de invasividad, ya que no se requiere de una laparotomía ni tampoco se realizarían punciones ni incisiones en los cuernos uterinos (Tabla 1).

\section{DISCUSIÓN}

En otros mamíferos se han establecido diferentes métodos de recuperación de embriones que van desde la utilización de sondas intrauterinas hasta la práctica de cirugía. En caninos, las investigaciones realizadas hasta el momento, han utilizado dos métodos de recuperación; el lavado del útero realizado por laparotomía o, la histerectomía y posterior lavado del útero para recuperar los embriones. En ambos casos se requeriría de cirugía para obtener los embriones.

Los tratamientos 1 y 2 tuvieron resultados de recuperación de estructuras similares ( 73 y $84.5 \%$ ) respectivamente, siendo estas TR muy buenas y similares a otros reportes de programas de transferencia de embriones (TE) (1-5). El T3 tuvo una TR del 58.3\% que es un poco baja. Las diferencias de los T1 y T2 respecto al T3 pudieron estar relacionadas con el flujo que se consigue durante el lavado y la recuperación de las estructuras.

En los tratamientos 1 y 2 , el flujo del medio de lavado es continuo mientras que en el tratamiento 3 se debe distender primero el útero y luego succionar para recuperar el medio. La dificultad de este método radica en que el succionar el medio de lavado, el catéter ejerce una presión negativa, generándose una adhesión del catéter a las paredes del útero, impidiendo que se recupere totalmente el medio de lavado. Este efecto posiblemente dificulta la recuperación de estructuras y explica por qué la tasa de recuperación es menor. También genera un inconveniente adicional si se quisiera implementar esta técnica en animales vivos, 
ya que habría un líquido remanente en el útero de la perra, que en ese momento estaría en diestro. Este líquido podría ser fuente de infección ya que los sistemas de evacuación (contractilidad uterina) y de defensa estarían disminuidos.

Tsutsui et al (2) evaluó la recuperación de embriones en tres perras de la raza Beagle, realizando histerectomía y lavando el útero con un medio de solución de Ringer con $20 \%$ de suero canino; en este experimento obtuvo una tasa de recuperación del 89.3\%. El mismo autor (3) reportó, tasas de recuperación de embriones de $97.1 \%$ por método de histerectomía en 16 animales y de $42.5 \%$ por laparotomía en 3 perras respecto al conteo de cuerpos luteos. Las tasas reportadas por este autor son similares a las encontradas en este experimento donde el T1 tuvo una TR del 73\% y el T2 del $84.5 \%$ con la diferencia que Tsusui (2) realizó una extracción completa del útero, mientras los métodos de los tratamientos 1 y 2 fueron realizados bajo laparotomía. Adicionalmente el T2 que tuvo la mejor TR, es un método que utilizó un catéter que evitó la incisión del útero.

Es de resaltar que el T3 a pesar de tener la menor tasa de recuperación entre los tres tratamientos realizados en este estudio, es superior respecto al reportado cuando se realizó laparotomía.

En otro trabajo realizado en zorros silvestres, se utilizaron 8 animales que fueron sacrificados para extraer el útero y luego recuperar los embriones por lavado (1). La tasa de recuperación fue de $66 \%$ basada en un promedio de cuerpos lúteos de 5.5. En este trabajo el autor también realizó la extracción completa del útero para luego ser lavado completamente sin especificar el método de lavado (1). La tasa obtenida por el autor (1) es inferior a la de los T1 y T2 y levemente superior a la del T3.

De acuerdo al estudio, la forma ideal de obtener embriones es a través del T3 donde la intervención quirúrgica no sería necesaria. Sin embargo, la tasa de recuperación es más baja posiblemente por el poco flujo que se obtiene durante el proceso de colecta de embriones. Es posible que esto se deba a la utilización del mismo catéter para distender y recuperar los embriones, sin que se tenga un sistema de dos vías como se trabaja en bovinos y equinos. Si se utilizara un catéter de doble vía donde se pueda mejorar el flujo al recuperar el fluido realizando el lavado por un lado y la recuperación del mismo por otro, se podría obtener una mejor tasa de recuperación.

Tsutsui et al (4) realizó otro trabajo en 2006 donde utilizó 10 hembras Beagle a las que les realizó histerectomía para obtener los úteros y recuperar los embriones lavando los úteros de la misma manera que lo hizo en los trabajos anteriores. En este trabajo reporto una TR del $88.6 \pm 6.8 \%$ con un rango de 36.4-100\% .Esta TR es muy similar a las obtenidas es este experimento pero tiene una diferencia en la desviación obtenidas por Tsutsui et al (2-4) la cual es alta comparada con la de los T1(7.30 \pm 2.4$)$, T2 $(8.45 \pm 1.9)$ y el T3 $(5.83 \pm 2.2)$.

Los pocos trabajos realizados en recuperación de embriones se han enfocado a técnicas de histerectomía que no son prácticas recomendables para la implementación en programas de transferencia de embriones para caninos, por ser muy invasivas y por poner en riesgo la capacidad reproductiva del animal. Las técnicas que se utilizaron en este trabajo están enfocadas a disminuir la afectación directa del útero conservando su capacidad reproductora. La técnica en la cual se utilizó un catéter transcervical (T3), logra este objetivo al no requerir cirugías durante el proceso de recuperación de embriones.

El grado de dificultad del experimento es mayor para el T3 por lo difícil de introducir un catéter transcervical sin cirugía. Sin embargo, el proceso de introducir un catéter a través del útero se realiza sin necesidad de cirugía en el caso de las inseminaciones transcervicales con la ayuda de una catéter noruego. Por consiguiente se considera que después de un proceso de aprendizaje, este procedimiento podría ser factible. Para el T2 el grado de dificultad esta dado por el hecho de realizar la cirugía y no por la introducción del catéter ni lavado que son 
más simples. En cuanto al grado de invasión es el normal de una cirugía ya que el útero solo recibe una punción. En contraste, en el T1 el grado de dificultad está dado por la cirugía y la incisión en la base del cuerno para ubicar el catéter Tomcat; esto también ocasiona un grado de invasión mayor y la posibilidad de generar adherencias en el útero.

Los métodos anteriormente descritos son eficientes según los resultados que se observan en los diferentes trabajos, éstos a su vez señalan que se puede seguir en el lavado y recuperación de embriones por vía transcervical y sin cirugía.

\section{Agradecimientos.}

Al doctor Luis Polo y a todos los colaboradores del departamento de Zoonosis de la Secretaria de Salud, por su disposición y colaboración. A la Universidad Nacional por la financiación parcial de este proyecto. Al equipo de International Breeders por su colaboración en esta investigación.

\section{REFERENCI AS}

1. Jalkanen L., Lindeberg H. Successful embryo transfer in the silver fox (Vulpes vulpes). Anim Reprod Sci 1998; 54: 139- 147.

2. Tsutsui T, Shimada K, Nishi M, K., Murao I. An experimental trial on embryo transfer in the dog. J Vet Med Sci 1989; 51: 797-800.

3. Tsutsui T, Hori T, Okazaki H. Transfer of canine embryo at various developmental stages recovered by hysterectomy or surgical uterine flushing. Theriogenology 2000; 63: 401-405.
4. Tsutsui T, Hori T, Endo S, Hayama A, Kawakami E. Intrauterine transfer of early canine embryos. Theriogenology 2006; 66: 1703-1705.

5. YeunHee Kim, Alexander J. Travis, Vicky N, Meyers-Wallen. Parturition Prediction and Timing of Canine Pregnancy. Theriogenology 2007; 68: 1177-1182. 